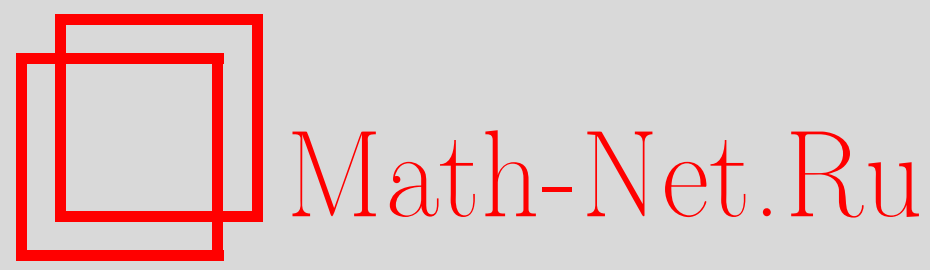

А. И. Данилушкин, Математическое моделирование и оптимизация процесса извлечения легкоплавкого наполнителя из металлического корпуса методом индукционного нагрева, Вестн. Сам. гос. техн. ун-та. Сер. Физ.-мат. науки, 1999, выпуск 7, 151-157

DOI: https://doi.org/10.14498/vsgtu219

Использование Общероссийского математического портала Math-Net.Ru подразумевает, что вы прочитали и согласны с пользовательским соглашением

http://www . mathnet.ru/rus/agreement

Параметры загрузки:

IP: 54.162 .85 .209

26 апреля 2023 г., 15:00:48

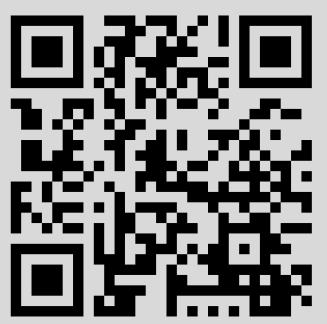




\section{А.И. Данилущкин}

\section{МАТЕМАТИЧЕСКОЕ МОДЕЛИРОВАНИЕ И ОПТИМИЗАЦИЯ ПРОЦЕССА ИЗВЛЕЧЕНИЯ ЛЕГКОПЛАВКОГО НАПОЛНИТЕЛЯ ИЗ МЕТАЛЛИЧЕСКОГО КОРПУСА МЕТОДОМ ИНДУКЦИОННОГО НАГРЕВА}

Рассматривается принии структурного моделирования и оптимального управления прочессом индукционного нагрева двух чилиндрических сопряженных физических неоднородных тел.

\section{1. Структурное моделирование процесса}

Для обработки неэлектрических материалов (плавки, сушки, вспенивания и т.д.) широко применяются реакторы и аппараты с индукционным нагревом. В силу известных преимуществ перед другими видами нагрева последний успешно внедряется в такие отрасли, как химическая, нефтеперерабатывающая, строительная индустрия, конверсионное производство. В оборонной промышленности разработан и внедряется новый метод утилизации боеприпасов, использующий индукционный нагрев как наиболее эффективный и экологически чистый способ извлечения взрывчатого вещества из корпусов снарядов, обеспечивающий возможность его повторного использования в строительстве, горнодобывающей и других отраслях производства.

В этой новой, весьма специфической области применения индукционного нагрева преимущества последнего позволяют создать конкурентоспособные установки стационарного и передвижного типа для утилизации боеприпасов. Широкая номенклатура расснаряжаемых изделий с различной геометрией корпусов обусловливает применение различных математических моделей для адекватного описания процессов теплопроводности в системе нагреваемых тел.

Качественное управление процессом возможно лишь при наличии полной информации о температурном режиме с учетом разнородности нагреваемьх материалов, реальной и обычно сложной конфигурации изделий, взаимодействия физических процессов различной природы. Однако получить столь подробную и полную информацию не представляется возможным. На практике часто приходится ограничиваться некоторым приближенным математическим описанием процесса, которое с определенной степенью точности, приемлемой для решения задач управления, позволяет обеспечить требуемые показатели качества синтезируемой системы.

В работе рассматривается в качестве приближенной математической модели процесса индукционного нагрева двух разнородных физически неоднородных сопряженных коротких цилиндров система линейных неоднородных уравнений второго порядка в частных производных вида

$$
\begin{gathered}
\frac{\partial \Theta_{1}(l, v, \varphi)}{\partial \varphi}=\frac{\partial^{2} \Theta_{1}(l, v, \varphi)}{\partial^{2}}+\frac{1}{l} \cdot \frac{\partial \Theta_{1}(l, v, \varphi)}{\partial}+\gamma \frac{\partial^{2} \Theta_{1}(l, v, \varphi)}{\partial v^{2}}+\Phi_{1}(l, v, \varphi) ; l \in\left[l_{1}, 1\right] v \in[0,1] \\
\frac{\partial \Theta_{2}(l, v, \varphi)}{\partial \varphi}=\frac{\partial^{2} \Theta_{2}(l, v, \varphi)}{\partial l^{2}}+\frac{1}{l} \cdot \frac{\partial \Theta_{2}(l, v, \varphi)}{\partial l}+\gamma \frac{\partial^{2} \Theta_{21}(l, v, \varphi)}{\partial v^{2}} ; \quad l \in\left[l_{1}, 1\right] ; \quad v \in[0,1] .
\end{gathered}
$$

Начальные условия

$$
\left.\Theta_{1}(l, \cup, \varphi)\right|_{\varphi=0}=\left.\Theta_{2}(l, v, \varphi)\right|_{\varphi=0}=0
$$

Граничные условия

$$
\begin{gathered}
\left.\frac{\partial \Theta_{1}(l, v, \varphi)}{\partial l}\right|_{l=1}=q_{1}(v, \varphi) ;\left.\quad \frac{\partial \Theta_{1}(l, v, \varphi)}{\partial l}\right|_{l=l_{1}}=\left.\beta^{\partial \Theta_{2}(l, v, \varphi)} \frac{}{\partial l}\right|_{l=l_{1}}, \quad v \in[0,1] ; \\
\Theta_{1}\left(l_{1}, \mathrm{v}, \varphi\right)=\Theta_{2}\left(l_{1}, \mathrm{v}, \varphi\right) ; \quad \frac{\partial \Theta_{2}(0, v, \varphi)}{\partial l}=0 ; \quad \forall v \in[0,1] \\
\frac{\partial \Theta_{1}(l, 0, \varphi)}{\partial v}=q_{2}(l, \varphi) ; \quad \frac{\partial \Theta_{1}(l, 1, \varphi)}{\partial v}=q_{3}(l, \varphi) ;
\end{gathered}
$$




$$
\Theta_{2}(l, 0, \varphi)=0 ; \quad \forall l \in\left[0, l_{1}\right] ; \quad \frac{\partial \Theta_{2}(l, 1, \varphi)}{\partial v}=q_{5}(l, \varphi) ; \quad \frac{\partial \Theta_{2}(l, 1, \varphi)}{\partial v}=q_{4}(l, \varphi) .
$$

Применяя к системе (1) - (4) cos-преобразование Фурье [1], получим :

$$
\begin{gathered}
\frac{\partial \bar{\Theta}_{1}(l, n, \varphi)}{\partial \varphi}=\frac{\partial^{2} \bar{\Theta}_{1}(l, n, \varphi)}{\partial^{2}}+\frac{1}{l} \cdot \frac{\partial \bar{\Theta}_{1}(l, n, \varphi)}{\partial}+\gamma\left[(-1)^{n} q_{3}(l, \varphi)-\right. \\
\frac{\partial \bar{\Theta}_{2}(l, n, \varphi)}{\partial \varphi}=\frac{\left.\partial_{2}(l, \varphi)-\pi^{2} n^{2} \bar{\Theta}_{1}(l, n, \varphi)\right]+\bar{\Phi}_{1}(l, n, \varphi)}{\partial l^{2}}+\frac{\partial \bar{\Theta}_{2}(l, n, \varphi)}{l \partial l}+\gamma\left[(-1)^{n} q_{5}(l, \varphi)-q_{4}(l, \varphi)-\pi^{2} n^{2} \bar{\Theta}_{2}(l, n, \varphi)\right] ; \\
\bar{\Theta}_{1}(l, n, 0)=\bar{\Theta}_{2}(l, n, 0)=0 ;\left.\quad \frac{\partial \bar{\Theta}_{1}(l, n, \varphi)}{\partial l}\right|_{l=1}=\bar{q}_{1}(n, \varphi) ; \\
\frac{\partial \bar{\Theta}_{1}(l, n, \varphi)}{\partial l}=\beta \frac{\partial \bar{\Theta}_{2}(l, n, \varphi)}{\partial l} ; \quad \bar{\Theta}_{1}(l, n, \varphi)=\bar{\Theta}_{2}(l, n, \varphi) ; \quad \frac{\partial \bar{\Theta}_{2}(0, n, \varphi)}{\partial l}=0 .
\end{gathered}
$$

Преобразуем (5) - (7) по Лапласу :

$$
\begin{aligned}
& \frac{d^{2} \overline{\bar{\Theta}}_{1}(l, n, p)}{d l^{2}}+\frac{d \overline{\bar{\Theta}}_{1}(l, n, p)}{l d l}-[p+A(n)] \overline{\bar{\Theta}}_{1}(l, n, p)+\overline{\bar{\Phi}}_{2}(l, n, p) \\
& \frac{d^{2} \overline{\bar{\Theta}}_{2}(l, n, p)}{d l^{2}}+\frac{d \overline{\bar{\Theta}}_{2}(l, n, p)}{l d l}-[p+A(n)] \overline{\bar{\Theta}}_{2}(l, n, p)+\overline{\bar{\Phi}}_{3}(l, p) .
\end{aligned}
$$

Здесь $\overline{\bar{\Phi}}_{2}(l, n, p)=\gamma(-1)^{n} q_{3}(p, l)-\gamma q_{2}(l, p)+\overline{\bar{\Phi}}_{1}(l, n, p)$;

$$
\begin{gathered}
\overline{\bar{\Phi}}_{3}(l, p)=\gamma(-1)^{n} q_{5}(l, p)-\gamma q_{4}(l, p) ; \quad A(n)=\pi^{2} n^{2} ; \\
\left.\frac{d \overline{\bar{\Theta}}_{1}(l, n, p)}{d l}\right|_{l=1}=\bar{q}_{1}(n, p) ;\left.\quad \frac{d \overline{\bar{\Theta}}_{1}(l, n, p)}{d l}\right|_{l=l_{1}}=\left.\beta \frac{d \overline{\bar{\Theta}}_{1}(l, n, p)}{d l}\right|_{l=l_{1}} ; \\
\overline{\bar{\Theta}}_{1}\left(l_{1}, n, p\right)=\overline{\bar{\Theta}}_{2}\left(l_{1}, n, p\right) ;\left.\quad \frac{d \overline{\bar{\Theta}}_{2}(l, n, p)}{d l}\right|_{l=0}=0 .
\end{gathered}
$$

Решение уравнения (8) запишем в виде [2]

$$
\overline{\bar{\Theta}}_{1}(l, n, p)=C_{1}(p+A(n)) I_{0}(l \sqrt{p+A(n)})+C_{2}(p+A(n)) K_{0}(l \sqrt{p+A(n)})+\overline{\bar{\Theta}}_{1}^{*}(l, n, p),
$$
где

$$
\overline{\bar{\Theta}}_{1}^{*}(l, n, p)=\int_{l_{1} \sqrt{p+A(n)}}^{l \sqrt{p+A(n)}} \xi\left[I_{0}(l \sqrt{p+A(n)}) K_{0}(\xi)-K_{0}(l \sqrt{p+A(n)}) I_{0}(\xi)\right] \overline{\bar{\Phi}}_{2}(\xi, n, p) d \xi .
$$

Пренебрегая, ввиду малости, зависимостью тепловых потоков с торцов цилиндра от радиальной координаты, представим решение уравнения (9) в виде

$$
\overline{\bar{\Theta}}_{2}(l, n, p)=C_{3}(p+A(n)) I_{0}(l \sqrt{p+A(n)})+\frac{\overline{\bar{\Phi}}_{3}(p)}{p+A(n)} .
$$

Обозначим в (12) $s=\sqrt{p+A(n)}$,

$$
\begin{aligned}
& B_{1}(l, n, p)=\int_{l_{1} s}^{l s} \xi K_{0}(\xi) \overline{\bar{\Phi}}_{2}(\xi, n, p) d \xi ; \\
& B_{2}(l, n, p)=\int_{l_{1} s}^{l_{s}} \xi I_{0}(\xi) \overline{\bar{\Phi}}_{2}(\xi, n, p) d \xi .
\end{aligned}
$$

Тогда решение (11) запишется в виде

$$
\overline{\bar{\Theta}}_{1}(l, n, p)=C_{1}(s) I_{0}(l s)+C_{2}(s) K_{0}(l s)+B_{1}(l, n, p) I_{0}(l s)+B_{2}(l, n, p) K_{0}(l s) .
$$


Постоянные интегрирования $C_{1}(s), C_{2}(s), C_{3}(s)$ найдем из граничных условий (10):

$$
\begin{gathered}
C_{1}(s)=\left[M_{1}(p, n) B_{2}(1, p, n)+M_{2}(p, n) B_{1}(1, p, n)-M_{3}(p, n) B_{1}\left(l_{1}, p, n\right)-\right. \\
\left.-M_{4}(p, n) B_{2}\left(l_{1}, p, n\right)+M_{5}(p, n) \overline{\bar{\Phi}}_{3}(p)+M_{6}(p, n) \overline{\bar{q}_{1}}(p, n)\right] \Psi^{-1}(p, n),
\end{gathered}
$$

где

$$
\begin{aligned}
& M_{1}(p, n)=K_{1}(s) s\left[K_{0}\left(l_{1} s\right) \beta I_{1}\left(l_{1} s\right)+I_{0}\left(l_{1} s\right) K_{1}\left(l_{1} s\right)\right] \\
& M_{2}(p, n)=I_{1}(s) s\left[K_{0}\left(l_{1} s\right) \beta I_{1}\left(l_{1} s\right)+I_{0}\left(l_{1} s\right) K_{1}\left(l_{1} s\right)\right] \\
& M_{3}(p, n)=\left[I_{1}\left(l_{1} s\right)+K_{1}\left(l_{1} s\right) \beta\right] I_{0}\left(l_{1} s\right) K_{1}(s) ; M_{4}(p, n)=\left[I_{0}\left(l_{1} s\right)+\beta K_{0}\left(l_{1} s\right)\right] K_{1}\left(l_{1} s\right) K_{1}(s) s \\
& M_{5}(p, n)=\beta K_{1}(s) K_{1}\left(l_{1} s\right) ; \quad M_{6}(p, n)=\left[K_{0}\left(l_{1} s\right) \beta I_{1}\left(l_{1} s\right)+I_{0}\left(l_{1} s\right) K_{1}\left(l_{1} s\right)\right] s \\
& \Psi(p, n)=s\left\{I_{1}\left(l_{1} s\right)\left[I_{0}\left(l_{1} s\right) K_{1}(s) \beta+K_{0}\left(l_{1} s\right) I_{1}(s) \beta-I_{0}\left(l_{1} s\right) K_{1}(s)\right]+I_{0}\left(l_{1} s\right) K_{1}\left(l_{1} s\right) I_{1}(s)\right\} \\
& C_{2}(n, p)=I_{1}(s) C_{1}(n, p)-\frac{I_{1}(s)}{K_{1}(s)} B_{1}(1, n, p)-\frac{q_{1}(n, p)}{s K_{1}(s)} \\
& C_{3}(n, p)=\left[\beta^{-1}-Q_{1}\left(l_{1}, p, n\right)\right] C_{1}(p, n)+Q_{2}\left(l_{1}, p, n\right) B_{2}(1, p, n)+ \\
& +Q_{3}\left(l_{1}, p, n\right) B_{1}(1, p, n)+Q_{4}\left(l_{1}, p, n\right) \bar{q}_{1}(p, n)-Q_{5}\left(l_{1}, p, n\right) B_{2}\left(l_{1}, n, p\right)-\beta^{-1} B_{1}\left(l_{1}, n, p\right)
\end{aligned}
$$

Здесь $\quad Q_{1}\left(l_{1}, n, p\right)=\frac{K_{1}\left(l_{1} s\right) I_{1}(s)}{\beta K_{1}(s) I_{1}\left(l_{1} s\right)} ; \quad Q_{2}\left(l_{1}, n, p\right)=\frac{K_{1}\left(l_{1} s\right)}{\beta I_{1}\left(l_{1} s\right)} ; \quad Q_{3}\left(l_{1}, n, p\right)=\frac{K_{1}\left(l_{1} s\right) I_{1}(s)}{s \beta K_{1}(s) I_{1}\left(l_{1} s\right)} ;$ $Q_{4}\left(l_{1}, n, p\right)=\frac{K_{1}\left(l_{1} s\right)}{\beta K_{1}(s) I_{1}\left(l_{1} s\right) s} ; Q_{5}\left(l_{1}, n, p\right)=\frac{K_{1}\left(l_{1} s\right)}{\beta I_{1}\left(l_{1} s\right)}$.

На рис.1 представлена структурная схема процесса теплопроводности двух сопряженных цилиндров с внутренними источниками тепла, распределенными по объему внешнего тепловыделяющего цилиндра. Структура составлена для граничных условий второго и четвертого родов и отражает взаимообусловленное формирование температурных полей в цилиндрах при нагреве внутренними источниками тепла.

Как следует из структурной модели процесса, температурные поля внешнего и внутреннего цилиндров формируются под действием внутренних источников тепла $\Phi(l, v, \varphi)$, тепловых потоков потерь $q_{1}(v, \varphi)$ с боковой поверхности внешнего цилиндра и тепловых потоков $q_{2}(l, \varphi), q_{3}(l, \varphi), q_{4}(l, \varphi), q_{5}(l, \varphi)$ соответственно с торцов внешнего и внутреннего цилиндров в условиях контактного теплообмена на границе сопряжения цилиндров. Влияние каждого из перечисленных факторов на температурное распределение в системе с учетом их взаимосвязи отражается введением в структурную схему процесса элементарных распределенных блоков, передаточные функции которых определены выше.

Функция $\Phi_{1}(l, v, \varphi)$ внутренних источников тепловыделения при индукционном нагреве с фиксированным параметром $\chi=\frac{R \sqrt{2}}{\Delta}$, где $\Delta$ - глубина проникновения тока в металл при обычных для индукционного нагрева допуцениях с достаточной для практических целей точностью, аптроксимируется выражением

$$
\Phi_{1}(l, v, \varphi)=W(v, \varphi) \cdot J(\chi, l),
$$

где $J(\chi, l)$ - функция распределения внутренних источников тепла по радиальной координате $l$, которая для рассматриваемого случая низкотемпературного нагрева ферромагнитной стали является нелинейной функцей, зависящей от температуры, свойств стали, напряженности поля и других параметров и может быть определена только численными методами. 


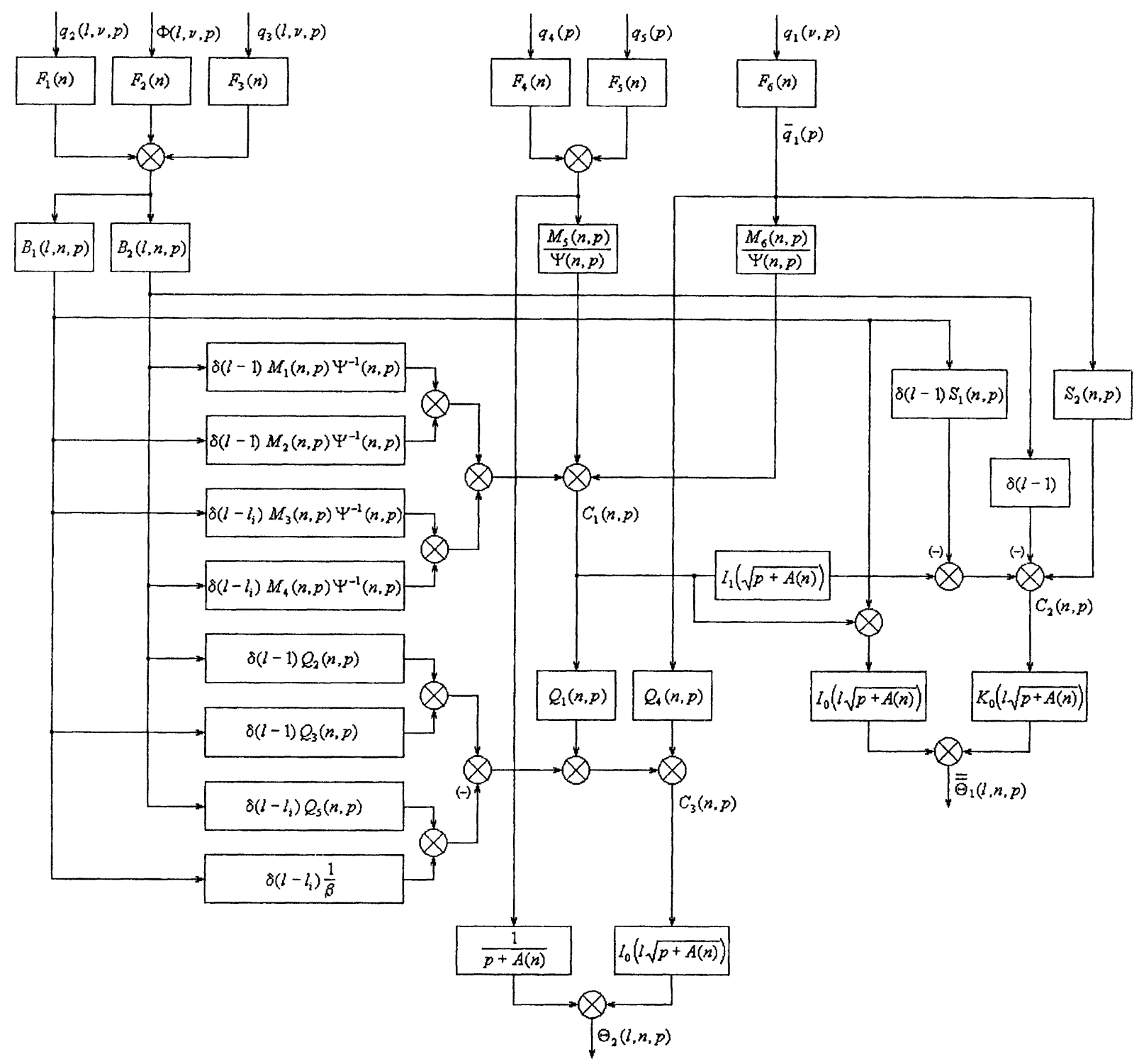

Р и с. 1. Структурная схема объекта управления

Однако при заданном технологической инструкцией диапазоне изменения температур перечисленные выше параметры и свойства стали можно считать постоянными. При соответствующем усреднении значений электромагнитных и теплофизических характеристик процесса функцию распределения мощности внутреннего тепловыделения можно представить в виде

$$
J(\chi, l)=\left\{\begin{array}{l}
\frac{12(l-\chi)^{2}}{(3+l)(1-\chi)^{3}}, \mathrm{p} \amalg 1-\chi \geq l \geq 1 ; \\
0, \cdot \mathrm{p} \amalg 0 \leq l \leq 1-\chi .
\end{array}\right.
$$

На основании структурной схемы легко может быть получена передаточная функция для температурного распределения $\bar{\Theta}_{1}(l, v, p), \bar{\Theta}_{2}(l, v, p)$ относительно любого из входных воздействий $\Phi(l, v, p), q_{1}(v, p), q_{2}(l, p), q_{3}(l, p), q_{4}(l, p), q_{5}(l, p)$.

Однако сложность выражения передаточной функции вызывает значительные трудности в решении конкретныт задач управления процессом. К тому же полученная передаточная функция отражает все второстепенные подробности динамики системы, которыми без ущерба для цели исследования можно пренебречь. Удовлетворительные результаты при решении задач анализа и синтеза замкнутых систем автоматического регулирования могут быть получены заменой сложных передаточных функций исследуемого объекта более простыми передаточными функциями эквивалентной математической модели. При этом задача приближения функций ставится следующим образом. Известна сложная передаточная функция, описывающая объект 
в целом. Требуется аппроксимировать эту передаточную функцию более простой дробнорациональной функцией с полиномами числителя и знаменателя более низкого порядка.

Для достижения этой цели достаточно получить близость изображений в области существенных частот. Совпадение изображений можно получить, если аптроксимировать точные логарифмические частотные характеристики объекта комбинацией соответствующих характеристик элементарных звеньев.

Точная передаточная функция объекта по каналу "мощность источников внутреннего тепловыделения - температура поверхности внешнего цилиндра", полученная на основании анализа структурной схемы процесса теплопроводности, имеет вид

$$
\begin{gathered}
W(l, v, p)=\frac{\Theta_{1}(l, v, p)}{\Phi(l, v, p)}=\sum_{n=1}^{\infty}\left\{B _ { 1 } ( l , n , p ) \left[\left(M_{2}(n, p)+M_{3}(n, p)\right) \times\right.\right. \\
\times\left(I_{1}(s) K_{0}(l s)+I_{0}(l s)\right) \delta(l-1)+S_{1}(n, p)\left(I_{0}(l s)+K_{0}(l s)\right) \delta(l-1)+ \\
\left.+I_{0}(l s)+K_{0}(l s)\right]+B_{2}(l, n, p)\left[\left(M_{1}(n, p)+M_{4}(n, p)\right) \times\left(I_{1}(s) K_{0}(l s)+\right.\right. \\
\left.\left.\left.+I_{0}(l s)\right) \delta(l-1)+K_{0}(l s) \delta(l-1)\right]\right\} \cos \pi n v .
\end{gathered}
$$

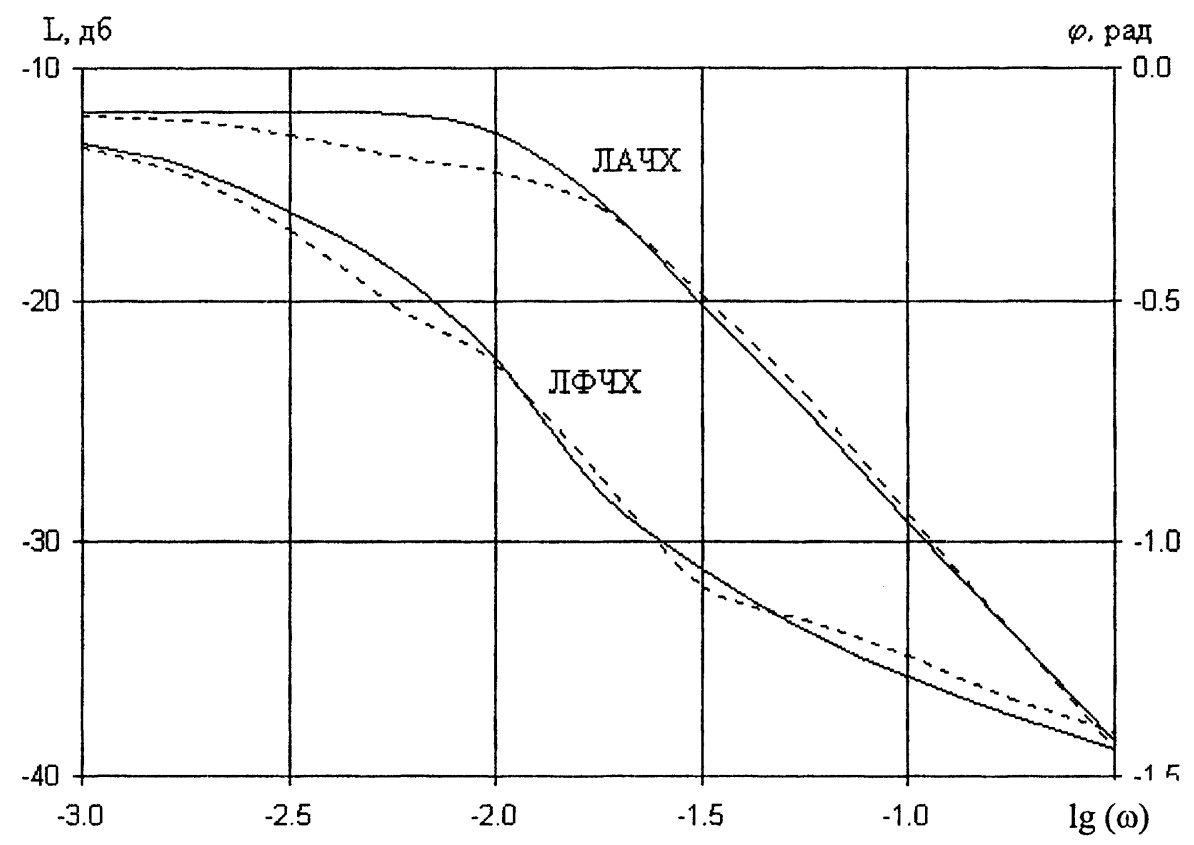

Р и с. 2. Аппроксимация динамических характеристик для внешнего цилиндра

На рис. 2 представлены полученные расчетным путем логарифмические амплитудные и фазовые частотные характеристики объекта. Расчетные характеристики имеют гладкий характер и монотонно убывают. Аппроксимированная передаточная функция имеет вид

$$
W_{a}(p)=\sum_{i=1}^{3} \frac{K_{i}}{T_{i} p+1} .
$$

Дальнейшее исследование динамики замкнутой системы автоматического регулирования осуществляется обычными методами теории автоматического регулирования на основе аппроксимированной передаточной функции

\section{2. Оптимальное управление процессом}

Технологический процесс извлечения продукта из корпуса снаряда состоит из двух стадий: нагрев корпуса до заданной температуры и выдержка при заданной температуре до момента полного выхода наполнителя из корпуса.

Для синтеза огтимального регулятора замкнутой САР примем допущение, которое в данной конкретной ситуации легко обосновывается: стенку корпуса можно с достаточной степе- 
нью точности считать теплотехнически тонким телом, что позволяет принять в качестве управляющего воздействия тепловой поток на поверхность извлекаемого продукта, однозначно связанный с мощностью $P(\varphi)$ источников внутреннего тепловыделения.

Тогда в качестве исходной модели можно рассматривать уравнение (2) для внутреннего цилиндра:

$$
\begin{gathered}
\frac{\partial \Theta_{2}(l, \varphi)}{\partial \varphi}=\mathbf{A}_{2} \frac{\partial^{2} \Theta_{2}(l, \varphi)}{\partial l^{2}}+\mathbf{A}_{1} \frac{\partial \Theta_{2}(l, \varphi)}{l \partial l} ; \\
\left.\frac{\partial \Theta_{2}(l, \varphi)}{\partial l}\right|_{l=l_{1}}=B_{1} U_{1}(\varphi) ;\left.\quad \frac{\partial \Theta_{2}(l, \varphi)}{\partial l}\right|_{l=0}=0 ;
\end{gathered}
$$

Здесь $\mathbf{A}_{2}$ - квадратная матрица, в которой по диагонали все элементы равны $a_{2}$, а остальные - нулевые; $\mathbf{A}_{1}$ - такая же матрица, в которой диагональные элементы равны $a_{2} \cdot e^{-1}$; $B_{1}=\frac{1}{\lambda}, a_{2}, \lambda$-соответственно коэффициенты температуропроводности и теплопроводности. Зададимся квадратичным критерием оптимальности

$$
\begin{aligned}
I=\frac{1}{2} \int_{0}^{1} \int_{0}^{1} \Theta_{2}(l, \varphi) \cdot \mathbf{S}_{f}(l, s) & \cdot \Theta_{2}\left(s, \varphi_{f}\right) d l d s+\frac{1}{2} \int_{0}^{\varphi_{f}} \int_{0}^{1} \int_{0}^{1}{ }_{2}^{T}(l, \varphi) \cdot \mathbf{F}(l, s, \varphi) \cdot{ }_{2}(s, \varphi) d l d s d \varphi+ \\
& +\frac{1}{2} \int_{0}^{1} \mathbf{U}^{T}(l, \varphi) \cdot \mathbf{E}_{1}(\varphi) \cdot \mathbf{U}_{1}(\varphi) d \varphi
\end{aligned}
$$

Здесь индекс «f» означает конечное состояние системы; первый член суммы отвечает за минимизацию среднеквадратичного отклонения температуры от заданной в конце процесса управления, второй член минимизирует отклонение температуры от заданного значения на протяжении всего процесса, что необходимо для удовлетворения ограничения по максимальной температуре $\Theta_{2}(1, \varphi)$ поверхности; третий член суммы характеризует затраты энергии на управление; $\mathbf{S}$ - координата заданной величины.

При выборе весовых функций $\mathbf{S}_{f}, \mathbf{F}, \mathbf{E}_{1}$, размерность которых $n \times n$, можно ограничиться несколькими значениями по координате $l$, больших некоторой величины $l_{2}$, так как в пределах цилиндра радиусом $l_{2}<l_{1}$ температура не достигает критических точек в процессе нагрева и выдержки.

Будем строить оптимальное управление с помощью принципа максимума [3]. Введем гамильтониан

$$
\begin{gathered}
H(l, \varphi)=\frac{1}{2} \int_{0}^{1} \Theta_{2}^{T}(l, \varphi) \mathbf{F} \Theta_{2}(s, \varphi) d s+\lambda \Theta_{2}(l, \varphi)\left[\mathbf{A}_{2} \ddot{\Theta}(l, \varphi)+\mathbf{A}_{1} \ddot{\Theta}(l, \varphi)\right] \\
H_{2}(\varphi)=\frac{1}{2} \mathbf{U}_{1}^{T}(l, \varphi) \mathbf{E}_{1}(\varphi) \mathbf{U}_{1}(\varphi)-\lambda^{T}(1, \varphi) \mathbf{A}_{2} \mathbf{B}_{1} \mathbf{U}_{1}(\varphi) \\
H_{1}(\varphi)=\frac{1}{2} \int_{0}^{1} \Theta_{2}\left(l, \varphi_{f}\right) \mathbf{S}_{f}(l, s) \Theta_{2}\left(s, \varphi_{f}\right) d s
\end{gathered}
$$

где $\lambda(1, \varphi)$ - вектор множителей Лагранжа .

Необходимые условия оптимальности для этого случая примут вид

$$
\frac{\partial H_{2}(l, \varphi)}{\partial \mathbf{U}_{1}(\varphi)}=\mathbf{E}_{1}(\varphi) \mathbf{U}_{1}(\varphi)+\mathbf{B}_{1}^{T} \mathbf{A}_{2}^{T} \lambda(1, \varphi)=0 .
$$

Обращая (23), получим

$$
\mathbf{U}_{1}(\varphi)=-\mathbf{E}_{1}^{-1}(\varphi) \mathbf{B}_{1}^{T} \mathbf{A}_{2}^{T} \lambda(1, \varphi) .
$$

Сопряженные переменные задаются уравнением 


$$
\frac{\partial \lambda(l, \varphi)}{\partial \varphi}=-\int_{0}^{1} \mathbf{F}(l, s) \Theta(s, \varphi) d s+\mathbf{A}_{1}^{T} \frac{\partial \lambda(l, \varphi)}{\partial l}-\mathbf{A}_{2}^{T} \frac{\partial^{2} \lambda(l, \varphi)}{\partial l}
$$

с граничными условиями

$\lambda\left(l, \varphi_{f}\right)=\int_{0}^{1} \mathbf{S}_{f}(l, s) \Theta\left(s, \varphi_{f}\right) d s ;-\mathbf{A}_{1}^{T} \lambda(0, \varphi)+\mathbf{A}_{2}^{T} \frac{\partial \lambda(0, \varphi)}{\partial l}=0 ;-\mathbf{A}_{1}^{T} \lambda(1, \varphi)+\mathbf{A}_{2}^{T} \frac{\partial \lambda(1, \varphi)}{\partial l}=0$.

Введем с помощью преобразования Рикатти матрицу $\mathbf{S}(l, s, \varphi)$ :

$$
\lambda(l, \varphi)=\int_{0}^{1} \mathbf{S}(l, s, \varphi) \Theta(s, \varphi) d s .
$$

Подставляя (25) в (24), получим для левой части (24)

$$
\frac{d \lambda(l, \varphi)}{d \varphi}=\int_{0}^{1}\left\{\dot{\mathbf{S}} \Theta(s, \varphi)+\mathbf{S}\left[\mathbf{A}_{2} \frac{\partial^{2} \Theta(s, \varphi)}{\partial s^{2}}+\mathbf{A}_{1} \frac{\partial \Theta(s, \varphi)}{\partial s}\right]\right\} d s .
$$

Правая часть (24) запишется в виде

$$
\int_{0}^{1}\left\{-\mathbf{F}(l, s) \Theta(s, \varphi)+\mathbf{A}_{1}^{T} \mathbf{S}_{r} \Theta(s, \varphi)-\mathbf{A}_{2}^{T} \mathbf{S}_{r r} \Theta(s, \varphi)\right\} d s .
$$

Индексы $r$ и $r r$ означают однократное и двукратное дифференцирование по координате $l$.

Преобразуя выражения (26), (27), получим окончательно

$$
\mathbf{S}_{\varphi}(l, s, \varphi)=-\mathbf{S}_{s s} \mathbf{A}_{2}-\mathbf{A}_{2}^{T} \mathbf{S}_{r r}+\mathbf{S}_{s} \mathbf{A}_{1}+\mathbf{A}_{1}^{T} \mathbf{S}_{r}+\mathbf{S}(l, 1, \varphi) \mathbf{A}_{2} \mathbf{B}_{1} \mathbf{E}_{1}^{-1} \mathbf{B}_{1}^{T} \mathbf{S}(l, 1, \varphi) \cdot \mathbf{F}(l, s, \varphi)
$$

с граничными условиями

$$
\mathbf{S}_{s}(l, 1, \varphi) \mathbf{A}_{2}-\mathbf{S}(l, 1, \varphi) \mathbf{A}_{1}=0, \mathbf{S}_{s}(l, 0, \varphi) \mathbf{A}_{2}-\mathbf{S}(l, 0, \varphi) \mathbf{A}_{1}=0
$$

и условием конечного состояния

$$
\mathbf{S}(l, s, \varphi)=\mathbf{S}_{f}(l, s) .
$$

Можно показать, что определённая таким образом матрица $\mathbf{S}$ является симметрической:

$$
\mathbf{S}(l, s, \varphi)=\mathbf{S}^{T}(l, s, \varphi) .
$$

Тогда для уравнения $\mathbf{U}(\varphi)$ оптимальный закон обратной связи принимает вид

$$
\mathbf{U}(\varphi)=-\mathbf{E}^{-1} \mathbf{B}_{1}^{T} \mathbf{A}_{2}^{T} \int_{0}^{1} \mathbf{S}(l, s, \varphi)(s, \varphi) d s .
$$

Значения матрицы $\mathbf{S}(l, s, \varphi)$ могут вычисляться заранее вне контура управления с помощью соотношений (28) - (31).

\section{БИБЛИОГРАФИЧЕСКИЙ СПИСОК}

1. Данилуикин А.И. Структурное моделирование процессов и систем управления для одного класса объектов индукционного нагрева// Вестн. Самар. техн. уњ-та. Вып. 5, 1998. С. 120-128.

2. Карташов Э.М. Аналитические методы в теории теплопроводности твёрдых тел. М.: Высш. школа, 1985.480 с.

3. Рей У. Методы управления технологическими процессами : Пер. с англ. М.: Мир, 1983. 368 с. 